\title{
Conocimiento de la flora y fauna predominante en el sitio prehispánico de San Andrés, valle de Zapotitán, departamento de La Libertad
}

\author{
The prevailing flora and fauna in the prehispanic site of San Andrés, \\ valle de Zapotitán, department of La Libertad
}

\section{Resumen}

En El Salvador existen escasas investigaciones sobre los recursos naturales y el rescate histórico de los diferentes usos de especies de la flora y la fauna que acompañaron la vida de las comunidades en sitios prehispánicos. Los escenarios sin duda han sido modificados a través de la historia. Ante esto surge la gran necesidad de sentar las bases sobre el estudio de la botánica y zoología aplicada a la Arqueología. Es por ello que se ha realizado una consulta bibliográfica sobre el sitio arqueológico San Andrés, a fin de brindar a la población salvadoreña datos interesantes sobre la Arqueobotánica y Arqueozoología. Para obtener información reciente y profundizar sobre la riqueza de este sitio se realizaron viajes de campo y así recabar información de los componentes florísticos y faunísticos de la actualidad y estudiar el museo del sitio para identificar elementos fito y zoomorfos.

\section{Palabras clave}

San Andrés - Historia; La Libertad, El Salvador - Historia; Sitios arqueológicos - El Salvador; Historia natural - El Salvador

\section{Abstract}

There are few investigations in El Salvador on natural resources and the historical rescue of the different uses of the fauna and flora species accompanying the life of those communities in prehispanic sites. Landscapes have undoubtedly been modified throughout history. Given this, the need arises for a study to lay the foundations on botany and zoology applied to archaeology. This gives way to a bibliographic research on the archaeological site of San Andrés, with the purpose to provide the Salvadorean society with interesting facts on Archaeobotany and Archaeozoology. In order to obtain recent information and deepen on the richness of this site, field trips were programmed so as to gather information on the current floral and animal components and therefore study the museum at the site to identify phytomorphic and zoomorphic elements.

\section{Keywords}

San Andrés - History; La Libertad, El Salvador - History; Archaelogical sites - El Salvador; Natural history - El Salvador 


\section{Introducción}

La estrecha banda geográfica que bordea la costa del Pacífico, desde El Salvador hasta el golfo de Guanacaste en costa Rica, actuó como frontera meridional de la Mesoamérica prehispánica. Se trató de una zona altamente poblada por sociedades de diferente signo cultural que recibieron bienes, ideas y personas procedentes de regiones tan lejanas como el centro de México. El oro, el algodón, así como otros tipos de productos tropicales fueron el principal reclamo de estas tierras para el mundo mesoamericano (Rovira, 2006-2008).

Por otra parte, las seis subáreas culturales de Mesoamérica contemplaron la sucesión de diferentes sociedades complejas que se desarrollaron de manera ininterrumpida desde el año 1500 a. C. hasta el 1519 d. C. Los diferentes períodos cronológicos en los que podemos segmentar la historia prehispánica de Mesoamérica son preclásico, clásico y postclásico (Rovira, 2006-2008).

Según Rovira (2006-2008) se han reportado pocos restos arqueobotánicos procedentes de excavaciones en El Salvador. Sheets (1982) menciona un olote carbonizado (zea mays) procedente de un contexto en Chalchuapa, correspondiente al preclásico tardío (aprox. 400 a. C.-300 d. C.). Los tallos y hojas de maíz, además de otras gramíneas y hojas, fueron preservados en impresiones en el sitio de Joya de Cerén (período clásico, 300-900 d. C.), como resultado de la erupción del cercano volcán Laguna Caldera. En el sitio correspondiente al posclásico temprano llamado Cihuatán, se han reportado fitolitos parecidos a los que caracteriza la corteza de mandioca (Manihot spp.) en muestras tomadas de los pisos, aunque la relación cronológica de estos depósitos (período posclásico o histórico) no está muy clara (McClung, 1985).

San Andrés es uno de los sitios arqueológicos más conocidos de El Salvador, desde su descubrimiento e intervenciones arqueológicas y su apertura como parque arqueológico en el año de 1996 hasta la actualidad. Perteneciente al periodo clásico, ha sido y sigue siendo de interés para nacionales, extranjeros, estudiantes e investigadores (Rovira, 2006-2008).

Este sitio fue una ciudad maya que controlaba los asentamientos más pequeños en el valle. En este lugar vivían gobernantes, artesanos, militares, sacerdotes, comerciantes, agricultores y muchas otras personas que formaban parte de la sociedad maya de esa época. Después que habitaron los mayas en San Andrés, Ilegaron grupos pipiles que vivieron por corto tiempo en la acrópolis (en línea, 2017).

Figura 1. Mapa de ubicación del sitio arqueológico San Andrés en El Salvador, Centroamérica. Fuente: Fundar.

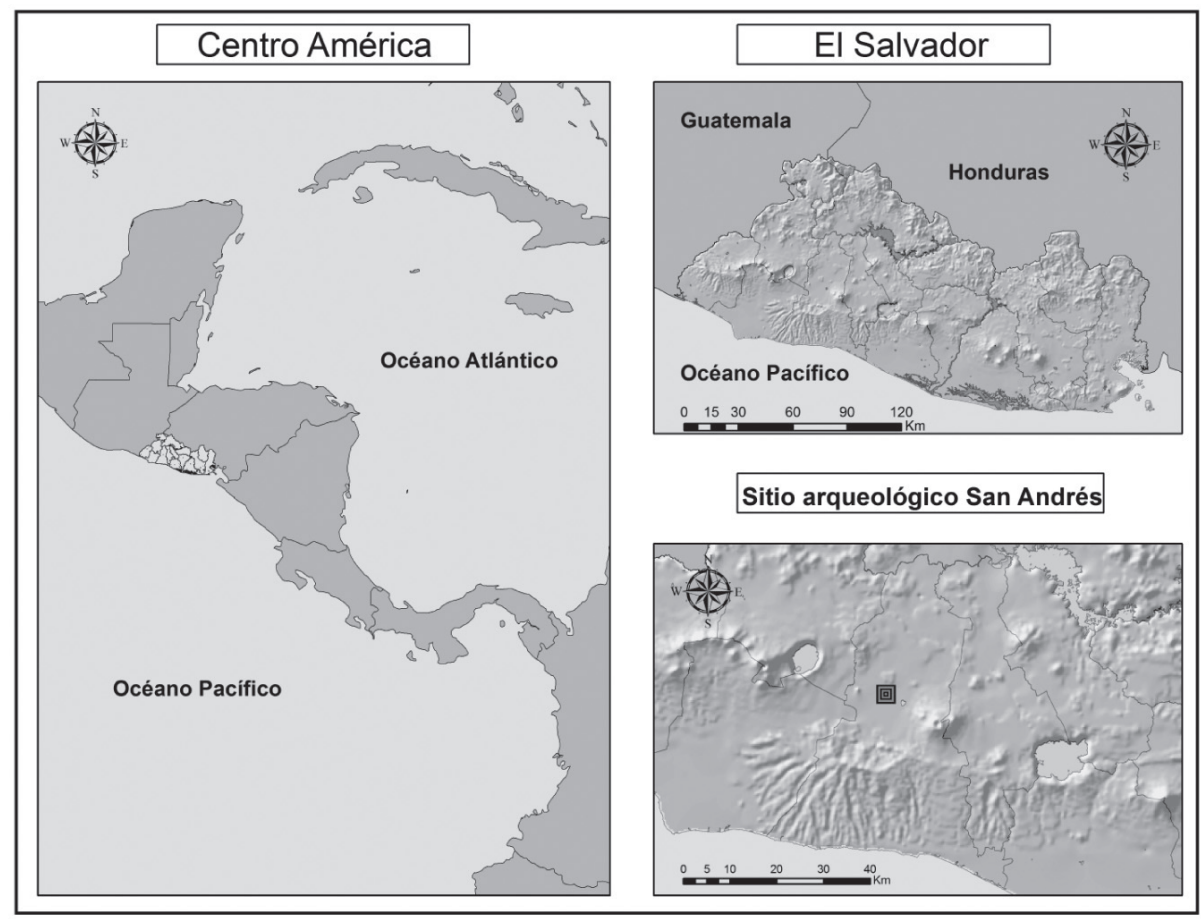




\section{Metodología}

Área de estudio

El Parque Arqueológico recibe su nombre debido a su ubicación en lo que anteriormente fue conocido como Hacienda San Andrés, está ubicado en la zona central del valle de Zapotitán ( $13^{\circ} 48^{\prime} 2^{\prime \prime} \mathrm{N},-89^{\circ} 23^{\prime} 21^{\prime \prime} \mathrm{W}$ ) [mapa1], en las riberas de los ríos Sucio y Agua Caliente, en el departamento de la Libertad, a una altura de 450 msnm (Fundar, n.d).

\section{Revisión bibliográfica}

Se realizó una búsqueda minuciosa de cada publicación que brinda información sobre el sitio arqueológico San Andrés, con el fin de obtener datos sobre hallazgos botánicos y zoológicos en el sitio, de tal manera que estos se sistematicen.

\section{Viajes de campo}

Las visitas de campo se realizaron entre los meses de febrero y abril, realizando caminatas libres en todo el terreno que actualmente está protegido para fotografiar y tomar muestras botánicas de aquellas plantas que estaban en estado fértil. De igual manera se anotó la presencia de recursos faunísticos actuales para evidenciar las modificaciones que ha sufrido esta zona.

Se visitó el departamento de Arqueología a fin de recabar información sobre excavaciones en el sitio arqueológico en investigación.

\section{Resultados}

Sitio arqueológico de San Andrés: retrospectiva histórica y bibliográfica

Las ruinas de San Andrés no eran bien conocidas de los arqueólogos antes de 1939 (Boggs, 1943). El mismo Boggs menciona que para los años cuarenta la lista de sitios no era muy grande y que Larde no menciona nada sobre este sitio, sin embargo, los propietarios de la hacienda San Andrés sí tenían conocimiento de su existencia.

Las investigaciones científicas en el sitio inician en 1940 con el proyecto dirigido por John Dimick, en el que llevan a cabo investigaciones en las estructuras 1, 2, 3 y 4 . Según el mismo Dimick, pequeñas excavaciones se llevaron a cabo para poder analizar el material cerámico y observar detalles arquitectónicos (Dimick, 1941).

En el año 1943 Stanley Boggs, que llega al país como fotógrafo de Dimick, inicia investigaciones en el sitio; y realiza excavaciones en la plaza, en el montículo 8 y cerca del montículo 5. "Se repararon hasta donde fue posible las paredes y pisos descubiertos, habiéndose empleado en esta labor como un tercio de diez a veinticinco hombres contratados para los trabajos" (Boggs, 1943). Estos trabajos de reparación de estructuras y pisos se llevaron a cabo revistiendo estos con una capa de cemento, con la única intención de conservarlos. Con el trabajo realizado por Boggs se elaboró el primer plano sobre la distribución del sitio.

Con estas primeras investigaciones se comienza una serie de excavaciones, tal es el caso que en la década de los años 40: Maurice Rise investigó La Campana; de 1977 a 1978 Jorge Mejía realizó excavaciones organizadas por el Museo Nacional de Antropología; en 1981 se inicia un proyecto para identificar vestigios arqueológicos dentro de las grandes haciendas por el biólogo Francisco Serrano, quien trabajó junto con el estadounidense Stanley Boggs (Fundar, n.d). En 1995 el arqueólogo estadounidense Paul Amaroli realizó excavaciones de sondeo en preparación para un nuevo museo; fue localizado un obraje de añil colonial, sepultado por la erupción de El Playón en noviembre de 1658 (Fundar, n.d); entre 1995 y 1997, Inez Verhaguen, Katherine Sampeck y Christopher Beagley realizaron investigaciones en el obraje de añil y excavaciones en La Campana. En 1997, se realizaron excavaciones en la pirámide principal (estructura 5, conocida como La Campana) y su entorno, las cuales fueron dirigidas por Christopher Beagley, con la participación de Jeb Card y Roberto Gallardo (Fundar, n.d); de 1997 a 1999, Brian R. McKee realizó investigaciones en la zona residencial de San Andrés; de 2005 a 2006, Paul Amaroli realizó excavación de un túnel en la Acrópolis. El departamento de Arqueología de la Secretaría de Cultura de la Presidencia (Secultura) realizó un levantamiento topográfico y excavaciones en el sitio (Secultura, 2011; Torres, 2010). Jorge Colorado, en 2011, hace una investigación sobre la astronomía de la estructura 7 del sitio. Nuevas investigaciones se están llevando a cabo en el sitio por parte del arqueólogo japonés Akira Ichikawa.

La existencia de grupos culturales en cualquier parte del planeta implica una interacción entre humanos y su entorno (medio ambiente). La utilización de plantas y animales para consumo, elaboración de herramientas o para rituales es un 
común denominador en todas las culturas. El emplazamiento de grupos humanos en los distintos hábitats llega a provocar una modificación del paisaje natural, que hace imperiosa la necesidad de profundizar estudios arqueobiológicos que puedan iluminar y reconstruir el paisaje histórico. Actualmente, mediante los estudios relacionados con la flora y la fauna, es muy difícil identificar las especies predominantes en el pasado distante.

Las excavaciones arqueológicas ejecutadas nos dan información de la historia que resguarda el Parque Arqueológico San Andrés. Este sitio se inicia desde el período preclásico y llega hasta el postclásico, aunque el auge lo tiene en el período clásico tardío (600 d. C.-900 d. C.) cuando las culturas mesoamericanas gozaban del esplendor que caracteriza a este período. San Andrés fue un centro rector, el cual, según algunos autores, tenía como aldeas tributarias algunas establecidas a lo ancho del valle de Zapotitán; sobresale una de ellas, que es lo que actualmente se conoce como Joya de Cerén. Este asentamiento salvadoreño es a su vez el único que se encuentra en la Lista de Patrimonio Mundial de la Organización de las Naciones Unidas para la Educación, la Ciencia y la Cultura (MacKee, 2011).

\section{Sitio San Andrés: elementos biológicos prehispánicos y actuales}

En épocas prehispánicas, el valle poseía una densa vegetación, rico en recursos naturales y con una gran biodiversidad. En este lugar se establecieron grupos mayas y posteriormente grupos pipiles provenientes del centro de México. Estos grupos humanos aprovecharon las fértiles tierras y sus recursos hídricos no solamente para irrigación, sino también para la pesca. De sus bosques también obtenían su alimentación, así como materiales para elaborar papel, extraían resinas con propósitos medicinales y fibras para diversos usos. Los mayas optimizaron los recursos agrícolas, cultivando una diversidad de plantas comestibles y medicinales. Estudios recientes han demostrado la alta producción de algunos bienes agrícolas en relación con el área cultivada dentro del valle, maximizando los recursos y obteniendo un excedente en la producción siempre y cuando las condiciones fueran las ideales, ya que el frágil balance, necesario para la producción agrícola, era conocido por los antiguos habitantes, así como lo es hoy en día (Secultura, 2011).
El sitio comprende una acrópolis, que es una plaza elevada con pirámides y casas de habitación en la parte superior. También hay una gran plaza en el lado norte donde se ubican otras pirámides, incluyendo una en forma de campana. Es posible que en este lugar hubiera un mercado donde se llevaba a cabo intercambio de bienes. En los alrededores, en chozas pequeñas, vivían los agricultores, quienes cosechaban los productos principales, como maíz, frijol y pipián. Todos los edificios estaban construidos con bloques de adobe, excepto dos estructuras construidas con bloques de toba volcánica, conocida localmente como talpetate.

A lo largo de las investigaciones realizadas en el sitio arqueológico poco o nada es lo que se habla sobre representaciones fitomorfas, son mucho más comunes las representaciones zoomorfas, asimismo, por el estado de conservación, no se tiene registro de ningún elemento orgánico procedente de San Andrés (Fig. 1).

En la investigación dirigida por Boggs se encuentran documentados piezas como cabezas de serpiente $y$ de loro labradas en piedra con espigas, una vasija con rasgos zoomorfos, incensarios con forma de serpiente cara zoomórfica desprendida de una vasija de piedra, y una cabeza grande de loro con espiga e incensarios con agarradera en forma de cabeza de serpiente (Boggs, 1943).

Podemos observar la frecuencia de rasgos zoomorfos, en especial de la serpiente; la aparición de representaciones de esta no se puede dejar a un lado, ya que, según lo que se puede leer en los escritos de Boggs, es el elemento más abundante.

Sobre de la tumba 2, se encontraron dos grandes cabezas de serpiente labradas en piedra, algunas conchas marinas, una de ellas con una perforación, lo cual hace presumir que podía ser utilizada como pendiente. Entre otros hallazgos menores se cuentan pitos de barro y fragmentos de figulinas. Los pitos poseen figuras globulares de cuerpos de animales con dos agujeros dactilares en la parte posterior. Las figuras de pájaros son las más corrientes y no muestran restos de pintura, pero en una figura de "búho" se descubren vestigios de un slip de color blanco mate. Tal vez el ejemplar más interesante, y a la vez el menos común, es una chirimía tubular con tres agujeros dactilares con una cabeza de lagartija aplicada como adorno (Boggs, 1943). 
Como podemos observar, en los escritos de Boggs se habla mucho sobre representaciones de animales en los artefactos encontrados, mas no leemos nada sobre plantas o frutos.

Algunas especies animales muy probablemente se vieron desplazadas por el mismo asentamiento de San Andrés; y acerca de las aldeas que se encontraban en todo el valle es bien sabido que donde existe una población (cuya economía y modo de subsistencia es evidentemente agrícola) hay un aprovechamiento de la tierra para la siembra. MacKee (2011) presenta un reporte detallado de sus estudios relacionados con la utilización de espacios en el valle de Zapotitán designados para el cultivo en el período protoclásico.

Estas áreas de cultivo se encontraron al occidente del parque arqueológico y yacían bajo depósitos de tierra blanca joven (conocida como TBJ) procedentes de la erupción del volcán de llopango (actualmente 535 d. C.). Este estudio aporta al conocimiento de la ocupación temprana del sitio, la especialización de la agricultura, así como a evidenciar la constante actividad volcánica que azotó a los sitios de El Salvador durante la época prehispánica.

La falta de investigaciones enfocadas en la flora y fauna de los sitios arqueológicos, y en específico de San Andrés, así como la escasa evidencia encontrada en las excavaciones, imposibilita (actualmente) concluir cuáles fueron las especies faunísticas y florísticas que durante la época prehispánica coexistían con mayor frecuencia. Sin embargo, fichas informativas de registro de excavaciones nos indican objetos que suponen la existencia de monos. En esas fichas también se encontró un aguijón de mantarraya, lo cual podría indicar el intercambio de especies animales entre los pobladores para con usos importantes en ritos y cotidianidades.

Sin embargo, en la biodiversidad actual registrada a lo largo de esta investigación, en las visitas de campo, identificamos 40 especies de plantas (tabla 1) y 5 especies de aves (tabla 2); y se tiene información, por parte de las personas que cuidan el parque, sobre la existencia de gatos de monte (Felis sp.) y cotuzas (Dasyprocta punctata) [Fig. 2].

Es interesante preguntarse cuál fue el proceso de tala y limpieza en el valle pre-San Andrés, qué especies vegetales y animales eran nativas del área y qué especies se vieron especialmente amenazadas con la presencia de grupos humanos. En cuanto a la evidencia prehispánica para investigar la flora y fauna en San Andrés, es primordial la cerámica, pues en la decoración de algunos tipos y sus variantes, o incluso en su forma, se aprecian representaciones tanto fito como zoomorfas.

Las representaciones zoomorfas son comunes en la cerámica del período clásico; y entre los elementos que se pueden observar en su diseño están aves, perros, peces y tortugas, entre otros. Es debido al conocimiento de la existencia de esta iconografía que se vuelve de interés realizar una revisión (de ser posible) de materiales culturales procedentes de los varios períodos de excavación en el Parque Arqueológico San Andrés.

Las representaciones fitomorfas parecen ser menos abundantes que las zoomorfas, para los materiales del periodo clásico en San Andrés; pero son conocidas en otros sitios cercanos, por ejemplo, en el ya mencionado patrimonio de la humanidad: Joya de Cerén.

\section{Materiales arqueológicos}

Entre los materiales culturales encontrados en San Andrés durante las excavaciones de entre 1940 y 1997 se incluyen cerámica Copador, cerámicos Guazapa, Chilama, Gualpopa policromo, Campana policromo, además de materiales líticos, cinco incensarios anulares con espigas (Boggs 1943a; Cobos y Sheets 1997; Dimick 1941). De los mencionados anteriormente, aquellos que demuestran evidencia de fauna son los pertenecientes a la cerámica Copador.

\section{Conclusiones}

La investigación arqueobotánica y la arqueozoológica en la actualidad tienen muchos vacíos de información en El Salvador por razones, como la falta de personal capacitado para llevar a cabo la recuperación e identificación de restos botánicos y zoológicos procedentes de sitios arqueológicos; y también por la falta de comunicación entre especialistas de la región que trabajen con datos procedentes de sitios en Mesoamérica.

En la actualidad, en nuestro país no hay conocimiento ni aplicación de metodologías para la conservación de material vegetal y animal antiguo, además no se cuenta con un laboratorio que reúna las condiciones para trabajar macropartículas provenientes de material antiguo. 
De las diferentes especies de plantas que en la actualidad se encuentran dentro del espacio comprendido por el parque arqueológico, muchas son introducidas (no nativas) para embellecer el entorno del parque, sin embargo, se encontraron especies nativas como cacao (Theobroma cacao), ceiba (Ceiba pentandra), bálsamo (Myroxylon balsamum var. pereirae), copinol (Hymenaea courbaril), que probablemente hayan sido parte de algún uso en tiempos prehispánicos.

\section{Recomendaciones}

Profundizar las metodologías e intensificar las investigaciones en esta área desconocida de los sitios arqueológicos.

Promover investigación con personal calificado a escala internacional, que permita iniciar un proceso de transferencia de conocimientos a nacionales que quieran estudiar la Arqueología y realizar análisis de material antiguo, como polen, que den un acercamiento de las especies vegetales con la cultura prehispánica.

Aunque es de todos bien conocido que en nuestro país no se cuenta con tecnología adecuada para el análisis de material antiguo, es importante que las instituciones responsables de estos saberes puedan establecer alianzas con otras instituciones de la región que fortalezcan los esfuerzos de investigación local.

\section{Referencias}

Boggs, S. (1943). "Notas sobre las excavaciones en la Hacienda 'San Andrés', Departamento de La Libertad". Tzumpame, 1 (especial).

Cobos, Rafael y Sheets, Payson (1997). "San Andrés y Joya de Cerén, Patrimonio de la Humanidad: encuentro con las huelas de nuestros antepasados". Volume 6 of Biblioteca Bancasa.

Colorado, J. (2013). "Alineación Astronómica de la Estructura 7 del Sitio Arqueológico San Andrés, El Salvador". Revista Digital Universitaria, 14 (5).

Dimick, J. (1941). Salvador. Carnegie Institution of Washintong, 40, 298-300.

Fundar (2011). "Fundar". Obtenido de http://www.fundar. org.sv/sanandre.html

Mcclung de Tapia, Emily (1985). "Investigaciones Arqueobotanicas en Mesoamérica y Centroamérica. Anales de Antropología". Vol. 22, n. ${ }^{\circ} 1$ (1985) McKee,

B. (2011). “Evidencia del uso agrícola del sitio San Andrés durante el período protoclásico". La universidad, 306-314.

Rise, M. (1940). First season archeological work at Campana San Andres, El Salvador. American Antropologis, 42.

Rovira Morgado, Rossend (2006-2008). "Mesoamérica: Concepto y Realidad de un Espacio Cultural". Antropología de América. Universidad Complutense de Madrid.

Secultura (2011). Sitio Arqueológico San Andrés. Datos sin publicar

Secultura en línea (mayo, 2017). http://www.cultura.gob.sv/ parque-arqueologico-san-andres/ Torres, U. (2010). "Investigaciones arqueológicas en la zona oriental de El Salvador" (Licenciatura). Universidad Tecnológica de El Salvador. 


\section{Anexos}

Tabla1. Listado de especies de plantas encontradas en sitio arqueológico

San Andrés y sus usos actuales

\begin{tabular}{|c|c|c|}
\hline Nombre científico & Nombre común & Uso \\
\hline Acacia sp. & izcanal & \\
\hline Agave angustifolia & agave & Artesanal y cultural \\
\hline Alpinia purpurata (Vieill.) K. Schum. & ginger & Ornamental \\
\hline Axonopus sp. & zacate & \\
\hline Callistemon lanceolatus Sweet & calistemo & Ornamental \\
\hline Cecropia peltata L. & guarumo & \\
\hline Ceiba pentandra (L.) Gaertn. & ceiba & Cultural \\
\hline Chrysobalanus icaco L. & icaco & Alimenticio \\
\hline Chrysophyllum cainitoL. & caimito & Alimenticio \\
\hline Cordia alliodora (Ruiz \& Pav.) Oken & laurel & Maderable \\
\hline Crescentia alata Kunth & morro & Alimenticio, artesanal \\
\hline Cyperus sp. & zacate & \\
\hline Delonix regia (Bojer ex Hook.) Raf. & árbol de fuego & Artesanal \\
\hline Desmodium sp. & pega-pega & \\
\hline Dypsis lutescens (H. Wendl.) Beentje \& J. Dransf. & palmera egipcia & \\
\hline Enterolobium cyclocarpum (Jacq.) Willd. & conacaste & Artesanal \\
\hline Euphorbia trigona Haw. & corcho & Ornamental \\
\hline Ficus indica L. & laurel de la India & \\
\hline Gliricidia sepium (Jacq.) Kunth ex Walp. & madrecaco & Alimenticio, energético y para sombra \\
\hline Hymenaea courbaril L. & copinol & Alimenticio, maderable \\
\hline \multicolumn{3}{|l|}{ Kallstroemia maxima (L.) Hook. \& Arn. } \\
\hline Lygodium venustum Sw. & crespillo & \\
\hline Mangifera indica L. & mango & Comestible \\
\hline Manilkara zapota (L.) P. Royen & níspero & Alimenticio \\
\hline Musa sp. & huerta & Alimenticio \\
\hline Myroxylon balsamum var. pereirae (Royle) Harms & bálsamo & Medicinal \\
\hline Oxalis frutescens L. & trebol de cuatro hojas & \\
\hline Paspalum sp. & zacate & \\
\hline Psidium guajava L. & guayabo & Alimenticio, artesanal y labranza \\
\hline Ricinus communis L. & higuero, higuerillo & \\
\hline Senna sp. & Senna & \\
\hline Sida acuta Burm. f. & escobilla & Artesanal \\
\hline Sideroxylon capiri subsp. tempisque (Pittier) T.D. Penn. & tenpisque & Alimenticio \\
\hline Solanum sp. & & \\
\hline
\end{tabular}




\begin{tabular}{|lll|}
\hline Syzygium cumini (L.) Skeels & cerezo de Belice & Alimenticio \\
\hline Tabebuia rosea (Bertol.) DC. & maquilishuat & Artesanal \\
\hline Terminalia catappa L. & almendro de río & Alimenticio \\
\hline Theobroma cacao L. & caco & Alimenticio, cultural \\
\hline Trichilia sp. & barre horno & Artesanal y construcción \\
\hline Tridax procumbens L. & hierba del toro & Medicinal \\
\hline
\end{tabular}

Tabla 2. Listado de especies de aves reportadas en el sitio arqueológico San Andrés

\begin{tabular}{|l|l|}
\hline \multicolumn{1}{|c|}{ Nombre científico } & \multicolumn{1}{c|}{ Nombre común } \\
\hline Campylorhynchus rufinucha & guacalchía \\
\hline Eumomota superciliosa & torogoz \\
\hline Icterus sp. & chiltota \\
\hline Piaya cayana & plátano asado \\
\hline Quiscalus mexicanus & zanate y clarinero \\
\hline
\end{tabular}

Figura 1. Fichas de registro de material fitomorfo en excavaciones arqueológicas en el sitio San Andrés, para el período prehispánico. (A-B) esfinge de cabeza de piedra esculpida, incorporando rasgos de jaguar con los de serpiente, (C-D) aguijón de mantarraya

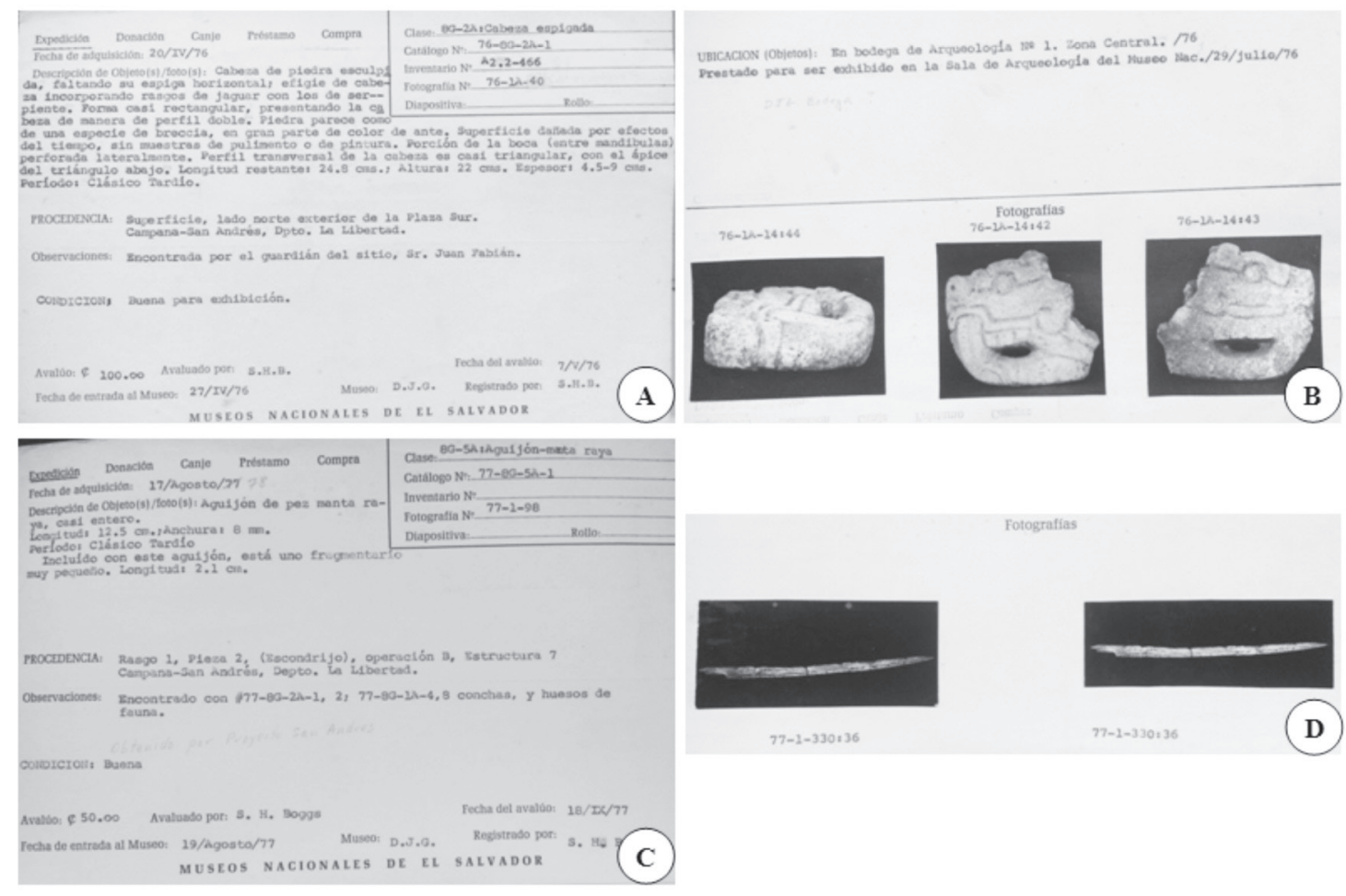


Figura 2. Especies de flora y fauna actuales en el sitio arqueológico San Andrés: (A) cacao [Theobroma caco], (B) morro [Crescentia alata], (C) torogoz [Eumomota superciliosa] y (D) zanate [Quiscalus mexicanus]

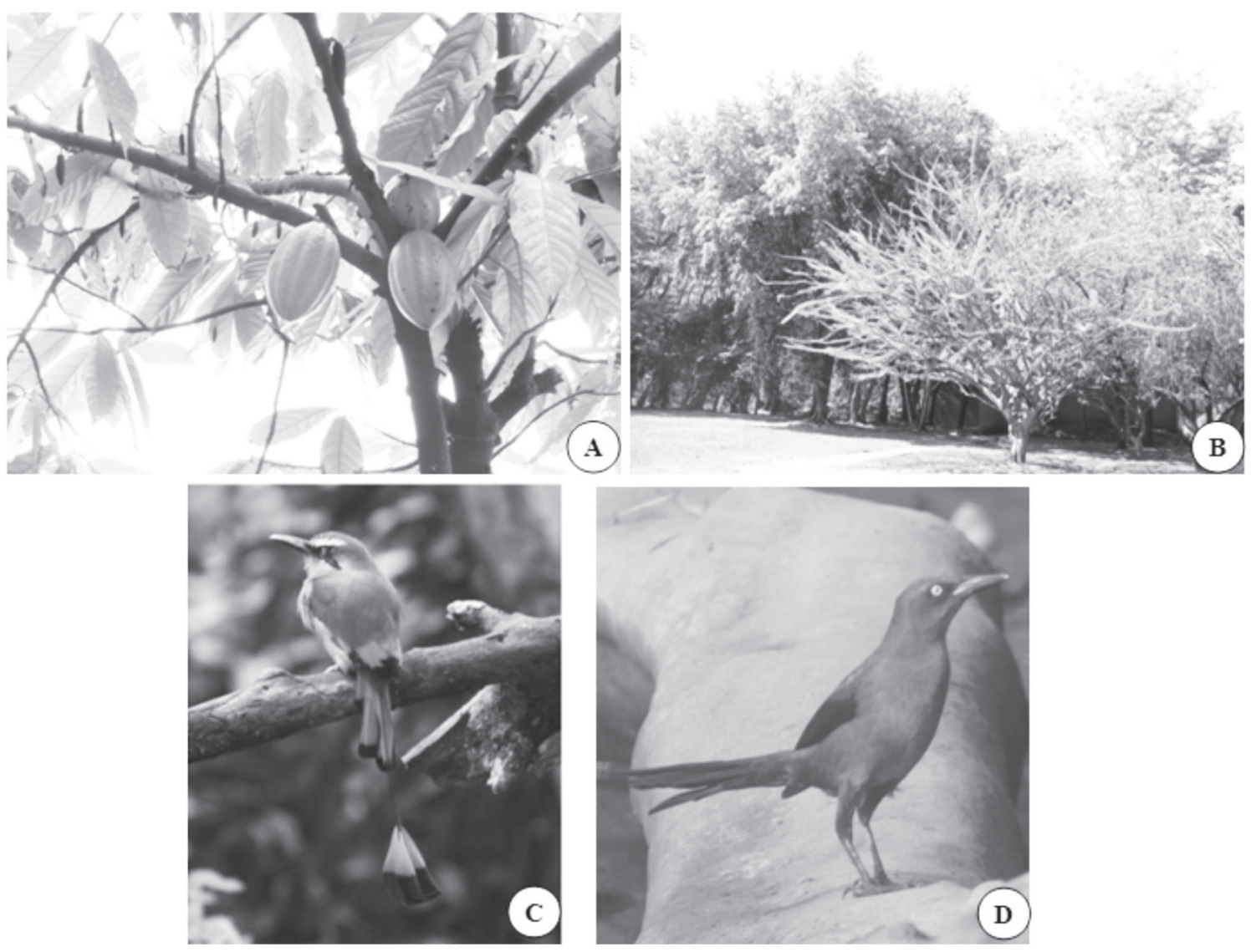

